\title{
PCR-Mediated Detection of Endophytic and Phytopathogenic Fungi from Needles of the Japanese Black Pine, Pinus thunbergii
}

\author{
Junichi Kihara*, Makoto Ueno, Sakae Arase \\ Faculty of Life and Environmental Science, Shimane University, Matsue, Japan \\ Email: ${ }^{*}$ j-kihara@life.shimane-u.ac.jp
}

Received 24 March 2015; accepted 14 April 2015; published 17 April 2015

Copyright (C) 2015 by authors and Scientific Research Publishing Inc.

This work is licensed under the Creative Commons Attribution International License (CC BY). http://creativecommons.org/licenses/by/4.0/

(c) (7) Open Access

\begin{abstract}
A specific and sensitive polymerase chain reaction (PCR) assay based on the internal transcribed spacer (ITS) region of rDNA sequences was developed to detect endophytic and phytopathogenic fungi from needles of the Japanese black pine, Pinus thunbergii. Sequences of the ITS regions of Lophodermium conigenum, Lecanosticta acicola, Pestalotiopsis neglecta, Rhizosphaera kalkhoffii, and Septorioides pini-thunbergii were compared, and each specific primer pair for these species was designed. First, the designed primer pairs were tested for their specificity to detect each species. A PCR product was amplified only each combination of species and its specific primer pair, confirming the specificity of the designed primer pairs. These primer pairs were also tested on DNA extracted from the needles of $P$. thunbergii. The PCR products were amplified not only in needles with lesions but also in healthy needles without symptoms. Furthermore, several endophytic and phytopathogenic fungi could be simultaneously detected from the same region in a needle. The PCR-mediated detection method developed in this study will be a valuable tool for the detection of the endophytic and phytopathogenic fungi, not only as a rapid diagnostic tool for early detection but also for monitoring variations in both the quality and quantity of the endophytic and phytopathogenic fungi in needles in Japanese black pines.
\end{abstract}

\section{Keywords}

Phytopathogenic Fungi, Endophytic Fungi, Pinus thunbergii, Japanese Black Pine, PCR-Mediated Detection

\footnotetext{
${ }^{*}$ Corresponding author.
}

How to cite this paper: Kihara, J., Ueno, M., \& Arase, S. (2015). PCR-Mediated Detection of Endophytic and Phytopathogenic Fungi from Needles of the Japanese Black Pine, Pinus thunbergii. Open Journal of Forestry, 5, 431-442. 


\section{Introduction}

The Japanese black pine (Pinus thunbergii Parl.), an evergreen species, is distributed along the seacoasts of Japan and South Korea. In addition, the Japanese black pine has been planted not only along seacoasts as a windbreak (Zhu et al., 2012) and to prevent soil erosion due to its resistance to salt (Townsend \& Kwolek, 1987) and various environmental stresses (Tsukahara et al., 1985), but also in public parks and gardens due to its beautiful appearance and toughness. Japanese black pines have been popular in Japan as both garden trees and bonsai (Chan, 2014). Many diseases of the Japanese black pine are known, such as Dothistroma needle blight caused by Dothistroma pini (Ito \& Zinno, 1972; Ito et al., 1975), brown spot needle blight caused by Lecanosticta acicola (teleomorph: Mycosphaerella dearnessii) (Suto \& Ougi, 1998; Seo et al., 2012), needle cast caused by Lophodermium spp. (Yamamoto et al., 1964; Sakuyama, 1993), Pestalotia disease caused by Pestalotiopsis spp. (Takahashi \& Kobayashi, 1998; Takahashi \& Kobayashi, 1999), Rhizosphaera needle blight caused by Rhizosphaera kalkhoffii (Tanaka \& Chiba, 1971), and sooty mold caused by Septorioides pini-thunbergii (synonym: Septoria pini-thunbergii) (Kaneko et al., 1989; Suto, 2000). It is difficult to distinguish between the diseases mentioned above because the diseases usually begin with the early symptom of yellowing and they are similar to each other, although the late symptoms of the diseases differ in terms of their characteristics. On the other hand, some of the fungi mentioned above have been considered to be endophytic fungi in Pinus spp. (Yoo \& Eom, 2012; Min et al., 2014; Qadri et al., 2014). The identification and detection of both endophytic and phytopathogenic fungi relies upon their culture-based morphological characteristics and on biochemical approaches. These procedures are time-consuming and require extensive knowledge of fungal taxonomy. Recently, a variety of molecular tools have been used to differentiate among fungal species. Among these, species-specific polymerase chain reaction (PCR) has emerged as a powerful tool for the identification and detection of phytopathogenic fungi, such as root rot pathogen Rhizopycnis vagum (Ghignone et al., 2003), collar rot pathogen Sclerotium rolfsii (Pravi et al., 2014), chestnut blight pathogen Cryphonectria parasitica (Popov et al., 2010), and pine needle pathogen Lophodermium spp. (Stenström \& Ihrmark, 2005). This paper reports the development of specific and rapid detection of endophytic and phytopathogenic fungi from the needles of Japanese black pines using PCR assay based on the internal transcribed spacer (ITS) region of rDNA sequences.

\section{Materials and Methods}

\subsection{Isolation of Endophytic and Phytopathogenic Fungi}

The needles of Japanese black pines with symptoms of disease were collected from the suburb around Shimane University, Matsue, Shimane Prefecture, Japan. We isolated endophytic and phytopathogenic fungi by two methods. The first, the surface sterilization method (Hata \& Futai, 1995), was used for isolation with minor modifications. Needles were cut and dipped in $70 \%$ ethanol for 1 min, surface sterilized for 5 min in a solution of $10 \%$ sodium hypochlorite solution (Wako Pure Chemical Industries, Osaka, Japan), rinsed in sterilized distilled water twice, and then dried on sterilized filter paper. Surface-sterilized samples were placed on potato dextrose agar (PDA) plates containing chloramphenicol $(20 \mu \mathrm{g} / \mathrm{ml})$ and incubated at $26^{\circ} \mathrm{C} \pm 1^{\circ} \mathrm{C}$ for $1-2$ weeks. In another method, needles washed with tap water were put on wet filter paper in a plastic box and then incubated at $26^{\circ} \mathrm{C} \pm 1^{\circ} \mathrm{C}$ in a growth chamber (LH-60FL3-DT, NK System, Osaka, Japan) for 1 to 3 weeks under a regime of $12 \mathrm{~h}$ of white light and $12 \mathrm{~h}$ of dark in order to form stroma of endophytic and phytopathogenic fungi on the needles. Spore masses formed on the needles were then picked up with a sterilized glass needle under a stereomicroscope (Goh, 1999; Choi et al., 1999), placed on PDA plates containing chloramphenicol (20 $\mu \mathrm{g} / \mathrm{ml})$, and incubated at $26^{\circ} \mathrm{C} \pm 1{ }^{\circ} \mathrm{C}$ for $1-2$ weeks.

\subsection{Molecular Identification of Phytopathogenic Fungi}

The identity of the isolates was confirmed by sequencing of the rDNA ITS region. Fungal isolates were grown on PDA medium for 1 week at $26^{\circ} \mathrm{C} \pm 1^{\circ} \mathrm{C}$. Mycelia were scraped and harvested in 1.5-ml Eppendorf micro tubes. DNA extraction was carried out using a Nucleo Spin Plant II kit (Macherey-Nagel, Düren, Germany) following the manufacturer's instructions, resuspended in $20 \mu \mathrm{l}$ of TE buffer, and stored at $-20^{\circ} \mathrm{C}$ until use. The universal primers ITS1 (5’-TCCGTAGGTGAACCTGCGG-3’) and ITS4

(5'-TCCTCCGCTTATTGATATGC-3') (White et al., 1990) were used to amplify the ITS-5.8S-ITS regions between the $18 \mathrm{~S}$ and $28 \mathrm{~S}$ nuclear rDNA. PCR reactions were performed using a Thermal Cycler GeneAtlas 
(Astec, Fukuoka Japan). The reaction mixture $(100 \mu \mathrm{l})$ contained about $20 \mathrm{ng}$ of the fungal genomic DNA, 0.5 $\mu \mathrm{M}$ of each primer, $0.2 \mathrm{mM}$ of each dNTP, $1 \times$ reaction buffer $\left(10 \mathrm{mM}\right.$ Tris-Cl, $\mathrm{pH} 8.3,1.5 \mathrm{mM} \mathrm{MgCl}_{2}, 50 \mathrm{mM}$ $\mathrm{KCl}$ ), and $2.5 \mathrm{U}$ of Taq DNA polymerase (TaKaRa, Osaka, Japan). The amplification cycle consisted of an initial heat denaturation step at $94^{\circ} \mathrm{C}$ for 2 min, followed by 25 cycles of $94^{\circ} \mathrm{C}$ for $30 \mathrm{sec}, 55^{\circ} \mathrm{C}$ for $30 \mathrm{sec}$, and $72^{\circ} \mathrm{C}$ for $30 \mathrm{sec}$, and a final extension at $72^{\circ} \mathrm{C}$ for $10 \mathrm{~min}$. The PCR products were electrophoresed in a $1 \%$ agarose gel in Tris-acetate-EDTA (TAE) buffer (40 mM Tris acetate, $1 \mathrm{mM}$ EDTA, pH 8.3), stained with ethidium bromide, destained in distilled water, and visualized under UV light (302 nm, UVP M-15V, UVP, Upland, CA). The PCR products were then excised and purified using the NucleoSpin Gel and PCR Clean-up kit (MachereyNagel) following the manufacturer's instructions. Sequencing reactions were performed using the BigDye ${ }^{\circledR}$ Terminator v3.1 Cycle Sequencing Kit (Life Technologies, Carlsbad, CA) according to the manufacturer's instructions. The DNA sequence analysis was performed on an ABI PRISM 310 genetic analyzer (Applied Biosystems, Foster City, CA). A computer analysis of the DNA sequence datawas performed using GENETYX ${ }^{\circledR}$ Mac (GENETYX, Tokyo, Japan). Comparisons between the DNA and the predicted aminoacid sequence as well as a phylogenetic analysis were carried out using the BLAST and CLUSTALW network programsat the DNA Data Bank of Japan (DDBJ, http://www.ddbj.nig.ac.jp).

\subsection{Primer Design and Primer Specificity Tests}

Sequences of each ITS region were aligned using CLUSTALW network programs at the DDBJ. Each specific primer pair within the ITS region was selected manually for species-specific detection. PCR reactions were performed by a Thermal Cycler GeneAtlas (Astec). The reaction mixture $(20 \mu \mathrm{l})$ contained $10 \mathrm{ng}$ of the fungal genomic DNA, $0.5 \mu \mathrm{M}$ of each primer, $0.2 \mathrm{mM}$ each dNTP, $1 \times$ reaction buffer $(10 \mathrm{mM}$ Tris-Cl, $\mathrm{pH}$ 8.3, $1.5 \mathrm{mM}$ $\mathrm{MgCl}_{2}, 50 \mathrm{mM} \mathrm{KCl}$ ), and $0.5 \mathrm{U}$ of Taq DNA polymerase (TaKaRa). The amplification cycle consisted of an initial heat denaturation step at $94^{\circ} \mathrm{C}$ for 2 min, followed by 25 cycles of $94^{\circ} \mathrm{C}$ for $30 \mathrm{sec}, 55^{\circ} \mathrm{C}$ for $30 \mathrm{sec}$, and $72^{\circ} \mathrm{C}$ for $30 \mathrm{sec}$; and a final extension at $72^{\circ} \mathrm{C}$ for $10 \mathrm{~min}$. PCR products were electrophoresed in a $1.5 \%$ agarose gel in TAE buffer, stained with ethidium bromide, destained in distilled water, and visualized under UV light (302 nm, UVP M-15V, UVP). An electrophoretogram was photographed using a gel documentation system (Print graph AE-6910FD, ATTO, Tokyo, Japan).

\subsection{Detection of Phytopathogenic Fungi from a Pine Needle}

The needles of Japanese black pines with or without symptoms of disease were collected from the suburb around Shimane University, Matsue, Shimane Prefecture, Japan. A detached needle from the Japanese black pine was washed in distilled water in order to remove microbial adhesion on the needle surface. DNA extraction from the needle was carried out using the NucleoSpin Plant II kit (Macherey-Nagel), resuspended in $50 \mu \mathrm{l}$ of TE buffer, and stored at $-20^{\circ} \mathrm{C}$ until use. The first PCR reactions were performed using primers ITS1 and ITS4 in order to amplify the ITS-5.8S-ITS rDNA fragment of the fungi as mentioned above. A second PCR reaction (nested PCR) was performed using each specific primer pair and $1 \mu \mathrm{l}$ of one-twentieth (1:20) diluted first PCR reaction mixture as a template DNA as mentioned above.

\section{Results}

\subsection{Isolation and Identification of Phytopathogenic Fungi}

We isolated 45 endophytic and phytopathogenic fungal candidates from $P$. thunbergii needles collected in Shimane prefecture, Japan (data not shown). The ITS regions of all of these fungi were sequenced. The frequent endophytic and phytopathogenic fungal candidates were Pestalotiopsis sp., L. conigenum, L. acicola, R. kalkhoffii, and S. pini-thunbergii. Thus, these five species were selected for further investigation (Table 1).

\subsection{Primer Pair Selections}

Sequences of ITS regions of L. conigenum, L. acicola, Pestalotiopsis neglecta, R. kalkhoffii, and S. pini-thunbergii (Table 1) were aligned by ClustalW (Figure 1), and specific forward and reverse primer pairs of oligonucleotides were designed from a non-consensus sequence of the alignment (Figure 1 and Table 2). In silico, primer pair specificity was evaluated by searching the DDBJ database. The BLAST search with the sequences 
Rk TCCGTAGGTGAACCTGCGGAAGGATCATTAAAG-AGTAA-GGGTC---TCCGGCC-CGA- 53

Sp TCCGTAGGTGAACCTGCGGAAGGATCATTACAG-AGTTCTGGGTCCT-TCGGGGC-CCG- 56

Lo TCCGTAGGTGAACCTGCGGAAGGATCATTAAAG-AATACAGGCTT---CGCGGCCGCTA- 55

Le TCCGTAGGTGAACCTGCGGAGGGATCATTAAAATACTGAAAGACCTCCCCTGGCCCCCGG 60

Pe TCCGTAGGTGAACCTGCGGAGGGATCATTATAG-------AGTTT---TCTAAAC----- 45

$$
* * * * * * * * * * * * * * * * * * * * * * * * * * * * * * * *
$$

Rk --------ACCTCCAACССTTTGTTGTTAAAACTA-CСTTGTTGCTTTGGCGGGAC 100 Sp ---------TCCTCCAACCCCTTGT---ATACATA-CCTCGTTGCTTCGGCGG-AC 98 LO ----------TATTCTCACCCTTTGTT---AACTACA-CTTTGTTGCCTTGGCG---- 95 Le GCCGGGGGAGTGATTTTCAAACCCTTGTG---AACTACAACTCTGTTGCTTCGGGGG--- 114 $\begin{array}{lr}\text { Le GCCGGGGGAGTGATTTTCAAACCCTTGTG---AACTACAACTCTGTTGCTTCGGGGG--- } & 114 \\ \text { Pe }---0 .-0 & 86\end{array}$ $* * * * * * * \quad * * * * * * * * *$

Rk CGTTCGGTCTCGAG-CGCAC-----CGGTTT-CTCCATTTCG------------AGGATT 141 Sp CAGCGCGTCGAGAGGCGCGCGTC--CGGCCC-CCTTGAACCG-------------GGGCT 142

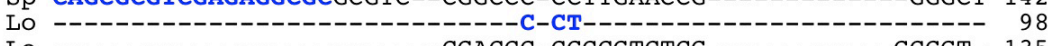
Le ---------------------CGACCC-CGCCGTCTCG-----------GCGGT- 135 Pe CTGCTCGgTGCACCTTACCTTGGAACGGCCTACCCTGTAGCGCCTTACCCTGGAACGGCT 146

Rk GG----TGAGCGCCCGCCA--GAGTCAAA--CCAAACTCTTGTATTAAACCA--GTCGTC 191 Sp GG----GGAGCGTCCGCCG--GAGGCCT---TCAAACTCTTGTTTGTAACGAT-GCAGTC 192 LO -------AGCGCCAGT----GGACCA-----AAACTCTTG-----AATCATTGCTGTC 135 Le --.--_-GGTGCTCCCGG--TGGCCATCTATCAAACTCT-GCATT--ACCTT-GC-GTC 180 Pe TACCCTGTAGCGGCTGCCGGTGGACTA---CCAAACTCTTGTTAT--TTTATTGTAATC 200
$* *$

Rk TGAGT---ATAAAATTTTAATTTAATTAAAACTTTCAACAACGGATCTCTTGGTTCTCGC 248 Sp TGA-----TCGAATATCAAATATTCTAAAACTTTCAACAACGGATCTCTTGGTTCTGGC 246 LO TGAGT---ACTATATAATAG-----TTAAAACTTTCAACAACGGATCTCTTGGTTCTGGC 187 Le GGAGTCTTATAAAGAATTAAA-----CAAAACTTTCAACAACGGATCTCTTGGTTCTGGC 235 Pe TGAGC---GTCTTATTTTAAT-AAGTCAAAACTTTCAACAACGGATCTCTTGGTTCTGGC 256 ** $* * * * * * * * * * * * * * * * * * * * * * * * * * * * * * * *$

Rk ATCGATGAagAaCGCAGCGAAATGCGATAAGTATGTGAATTGCAGAATTCAGTGAATCA 308 Sp ATCGATGAAGAACGCAGCGAAATGCGATAAGTAATGTGAATTGCAGAATTCAGTGAATCA 306 LO ATCGATGAAGAACGCAGCGAAATGCGATAAGTAATGTGAATTGCAGAATTCAGTGAATCA 247 Le ATCGATGAAGAACGCAGCGAAATGCGATAAGTAATGTGAATTGCAGAATTCAGTGAATCA 295

Pe ATCGATGAAGAACGCAGCGAAATGCGATAAGTAATGTGAATTGCAGAATTCAGTGAATCA 316 $* * * * * * * * * * * * * * * * * * * * * * * * * * * * * * * * * * * * * * * * * * * * * * * * * * * * * * * * * * * *$

Rk TCGAATCTTTGAACGCACATTGCGCCCCTTGGTATTCCGAGGGGCATGCCTGTTCGAGCG 368 Sp TCGAATCTTTGAACGCACATTGCGCCCCTTGGCATTCCGAGGGGCATGCCTGTTCGAGCG 366 Lo TCGAATCTTTGAACGCACATTGCGCCCCCTGGCATTCCGGGGGGCATGCCTGTTCGAGCG 307

Le TCGAATCTTTGAACGCACATTGCGCCCCGTGGTATTCCGCGGGGCATGCCTGTTCGAGCG 355

Pe TCGAATCTTTGAACGCACATTGCGCCCATTAGTATTCTAGTGGGCATGCCTGTTCGAGCG 376 TCATTACACCACTCAAGCACTGCTTGGTATTGGGCA-CC-CGTCCGCCGAAAGGCGGGC- 425 Sp TCATTACAACCCTCAAGCTCTGCTTGGTGTTGGGC--CT-CGTCCCCC----CGCGGAC- 418 LO TCATTACAACCCTCAAGCTCTGCTTGGTATTGGGC---T-CGCCCCGTA------GGGC- 356 Le TCATTTCACCACTCAAGCCTGGCTTGGTATTGGGCG-TCGCGGCCTCC---------GC- 404

Pe TCATTTCAACCCTTAAGCCTAGCTTAGTGTTGGGAGACTACTGCTTTTA-----CTAGCT 431

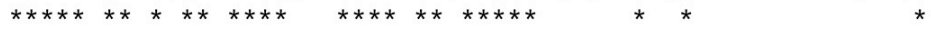

Rk ---GTGCCTCGAAGACCTCGGCGG-GGCCTAACCGGCT-TCGGGCGTAGTAGAGTTAAAT 480 Sp ---GTGCCTCAAAATCATCGGCGGTGGCGT--CTTGCC-TCAAGCGTAGTAAAAT----T 468 LO ---CTGCCTCAAAATCAGTGGCGGCCAC-TGTCCGACCCTTCAGCGCAGTACTAC----T 408 Le ---GCGCCTCAAAGTCTCCGGCTG-AGC--AGTCCGTCTCCGAGCGTTGTGACAT----- 453 Pe GTAGCTCCTGAAATACAACGGCGG-ATCTGCGATATCCTCTGAGCGTAGTAATTT----T 486 $* * * * * * * * * * * * * *$

Rk CAAAACGTCTTATA----AGTCTGGTTAGAACCCATTGCCGTA-AAACCTTTTTATTT-- 533 Sp CTTCTCGCTTTGGA----GGTTGGG---GCGCCCCCCGCCGGACGAACCTTTATACTTCT 521 LO CGCCGCTCGTAGGA----GGATGGG---AAGCC----GTTATACAACCCCCACCATA--- 454 Le --TTTCGCTAGGGA----GTTCGCGTC--TGCC-GCGGCCGTTAAATCATTAACACCA-- 502 Pe TATCTCGCTTTTGACTGGAGTTGCA---GCGTCTTTAGCCGCTAAATCCCCCA-ATTT-- 540

$$
\text { * * * * * * * * }
$$

Rk -TCTA-GGTTGACCTCGgATCAGGTAGGGATACCCGCTGAACTTAA

Sp ATCAA-GGTTGACCTCGGATCAGGTAGGGATACCCGCTGAACTTAAGCATATCAATAAGC 580 LO --CAA-GGTTGACCTCGGATCAGGTAGGGATACCCGCTGAACTTAAGCATATCAATAAGC 511 Le ---AA-GGTTGACCTCGGATCAGGTAGGGATACCCGCTGAACTTAAGCATATCAATAAGC 558

Pe -TTAATGGTTGACCTCGGATCAGGTAGGAATACCCGCTGAACTTAAGCATATCAATAAGC 599

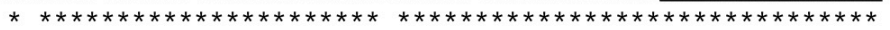

Rk GGAGGA 597

SP GGAGGA 586

LO GGAGGA 517

Le GGAGGA 564

Pe GGAGGA 605

Figure 1. Nucleotide sequence alignment of ITS-5.8S-ITS rDNA amplified by PCR using the primers ITS1 and ITS4 in five phytopathogenic fungi. Identical nucleotide positions among the five phytopathogenic fungi are indicated by asterisks. Bold type marked in blue indicates the primer sequence of each species (Table 2). Sequences in boxes (upper left and lower right) indicate the primer sequences of ITS1 and ITS4, respectively. Rk, R. kalkhoffii; Sp, S. pini-thunbergii; Lo, L. conigenum; Le, L. acicola; Pe, P. neglecta. 
Table 1. Possible identities of endophytes isolated from $P$. thunbergii needles based on the GenBank database.

\begin{tabular}{|c|c|c|c|c|c|}
\hline \multirow{2}{*}{ Possible fungal identity } & \multirow{2}{*}{ Isolate } & \multirow{2}{*}{$\begin{array}{l}\text { GenBank } \\
\text { Acc. No. }\end{array}$} & \multicolumn{3}{|c|}{ BLAST match with high similarity } \\
\hline & & & Definition & GenBank Acc. No. & Similarity (\%) \\
\hline Lophodermium conigenum & A08 & LC033959 & L. conigenum & FJ861972 & $476 / 478(99 \%)$ \\
\hline Lophodermium conigenum & A10 & LC033960 & L. conigenum & FJ861976 & $477 / 478(99 \%)$ \\
\hline Lecanosticta acicola & A03 & LC033961 & L. acicola & HМ367708 & $525 / 525(100 \%)$ \\
\hline Lecanosticta acicola & A04 & LC033962 & L. acicola & HM367708 & $525 / 525(100 \%)$ \\
\hline Pestalotiopsis neglecta & A06 & LC033963 & Pestalotiopsis sp. & KF313103 & $566 / 566(100 \%)$ \\
\hline Pestalotiopsis neglecta & J01-2 & LC033964 & Pestalotiopsis sp. & KF313103 & $566 / 566(100 \%)$ \\
\hline Rhizosphaera kalkhoffii & TEC01-1 & LC033965 & Rhizosphaera sp. & HM595558 & $555 / 558(99 \%)$ \\
\hline Rhizosphaera kalkhoffii & TEC01-2 & LC033966 & Rhizosphaera sp. & HM595558 & $555 / 558(99 \%)$ \\
\hline Septorioides pini-thunbergii & TEC01-3 & LC033967 & S. pini-thunbergii & KF251243 & $543 / 543(100 \%)$ \\
\hline Septorioides pini-thunbergii & YA02-1 & LC033968 & S. pini-thunbergii & KF251243 & $543 / 543(100 \%)$ \\
\hline
\end{tabular}

Table 2. PCR primers used in this study.

\begin{tabular}{ccc}
\hline Species & Primer name & Sequence (5' to 3') \\
\hline Lophodermium conigenum & Lo1F & TGCCTTGGCGCCTAGCGCCA \\
Lecanosticta acicola & Lo1R & CTCCTACGAGCGGCGAGTAG \\
Le1F & GCTCCCGGTGGCCATCTATC \\
Pestalotiopsis neglecta & Le1R & GAACTCCCTAGCGAAAATGT \\
Pe1F & Pe1R & CTCGGTGCACCTTACCTTGG \\
Rhizosphaera kalkhoffii & Rk1F & AAAGACGCTGCAACTCCAGT \\
Septorioides pini-thunbergii & Rk1R & TCTCGAGCGCACCGGTTTCT \\
& Sp1F & CCAGACTTATAAGACGTTTTGATTT \\
Pinus thunbergii & Sp1R & ACCAGCGCGTCGAGAGGCGC \\
(RuBisCO large subunit) & rbcLF & AACCTCCAAAGCGAGAAGAAT \\
\hline
\end{tabular}

of the primer pairs Lo1F/Lo1R, Le1F/Le1R, Pn1F/Pn1R, Rk1F/Rk1R, and Sp1F/Sp1R as a query showed the fungal sequences most similar to L. conigenum, L. acicola, Pestalotiopsis sp., R. kalkhoffii, and S. pini-thunbergii, respectively, suggesting that each primer pair was specific for detecting each phytopathgenic fungus by PCR amplification of the ITS-5.8S-ITS region (data not shown).

\subsection{Primer Specificity Test}

To confirm the specificity of the primer pairs, PCR was carried out using each primer pair and the genomic DNA of each phytopathogenic fungus as a template (Figure 2). No amplification was detected using the rbcLF/ rbcLR primer pair to target the RuBisCO large subunit gene of the Japanese black pine with each template of genomic DNA in all phytopathogenic fungi (Figures 2(a)-(h)), confirming that the rbcLF/rbcLR primer pair does not interact with the fungal genomic DNA used in this study. On the other hand, amplification was detected using the ITS1/ITS4 primer pair with each template of genomic DNA in all fungi (Figures 2(a)-(h)), indicating 
(a)

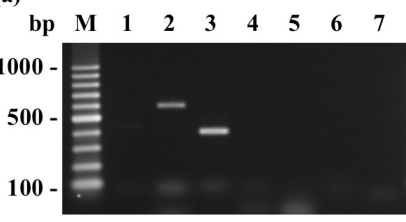

(c)

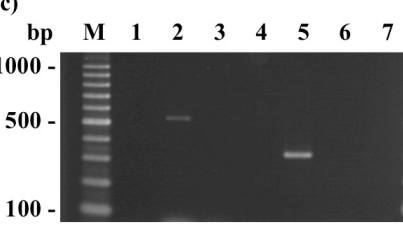

(e)

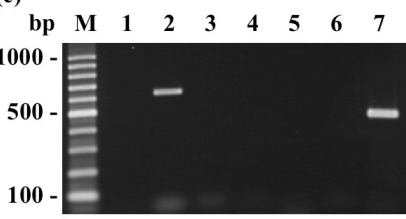

(g)

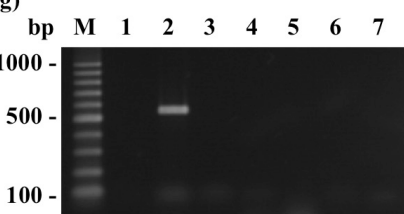

(b)

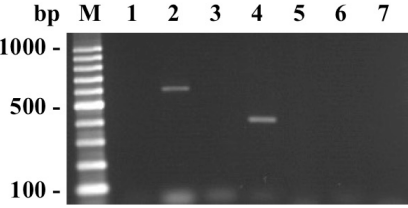

(d)

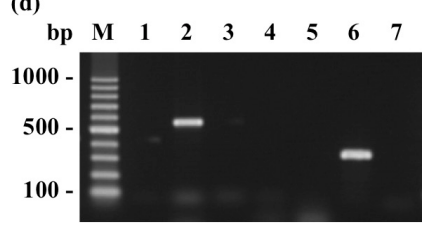

(f)
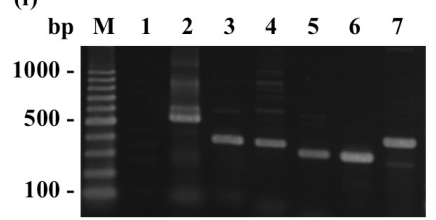

(h)
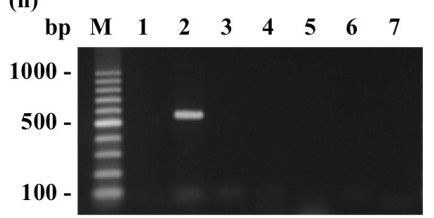

Figure 2. Primer specificity test. (a) R. kalkhoffii; (b) S. pini-thunbergii; (c) L. conigenum; (d) L. acicola; (e); P. neglecta (f) R. kalkhoffii + S. pini-thunbergii + L. conigenum + L. acicola + P. neglecta; (g) Cladosporiumsp.; (h) Diaporthe sp.. Lane 1, rbcLF/rbcLR (RuBisCO large subunit gene of $P$. thunbergii); lane 2, ITS1/ITS4 (fungal ITS-5.8S-ITS rDNA); lane 3, Rk1F/Rk1R (R. kalkhoffii); lane 4, Sp1F/Sp1R (S. pini-thunbergii); lane 5, Lo1F/Lo1R (L. conigenum); lane 6, Le1F/Le1R (L. acicola); lane7, Pe1F/Pe1R (P. neglecta). M, 100 bp DNA ladder marker.

that the ITS1/ITS4 primer pair could be used to generally amplify the ITS-5.8S-ITS rDNA of fungi as a universal primer. On the other hand, the use of each specific primer pair was successful for detecting the target species; only the primer pair Rk1F/Rk1R successfully amplified the target DNA in the predicted single band of 393 bp from R. Kalkhoffii (Figure 2(a), lane 3); only the primer pair Sp1F/Sp1R successfully amplified the target DNA in the predicted single band of 390 bp from S. pini-thunbergii (Figure 2(b), lane 4); only the primer pair Lo1F/Lo1R successfully amplified the target DNA in the predicted single band of 337 bp from L. conigenum (Figure 2(c), lane 5); only the primer pair Le1F/Le1R successfully amplified the target DNA in the predicted single band of 333 bp from L. acicola (Figure 2(d), lane 6); only the primer pair Pn1F/Pn1R successfully amplified the target DNAin the predicted single band of 431 bp from $P$. neglecta (Figure 2(e), lane 7); and furthermore, all amplification products were obtained using each primer pair with a DNA mixture of $R$. kalkhoffii, S. pini-thunbergii, L. conigenum, L. acicola, and P. neglecta (Figure 2(f), lanes 2 - 7). No amplification product was obtained using any of the primer pairs except ITS1/ITS4 as a fungal ITS universal primer with each template of genomic DNA from Cladosporium sp. (Figure 2(g)) and Diaporthe sp. (Figure 2(h)). These results suggested that each specific primer pair would be fit to detect each endophytic and phytopathogenic fungus even if several endophytic and phytopathogenic fungi were present in the same needle from a Japanese black pine.

\subsection{Detection of Endophytic and Phytopathogenic Fungi from a $P$, thunbergii Needle}

We evaluated whether any endophytic or phytopathogenic fungi could be detected from a needle (Figure 3). Amplification products were obtained using the rbcLF/rbcLR primer pair to target the RuBisCO large subunit gene in sample A and sample B but not in sample C (Figure 3(b)). These results indicate that the RuBisCO large subunit gene would be detectable from the greening region of a needle, whereas it would not be detectable from 


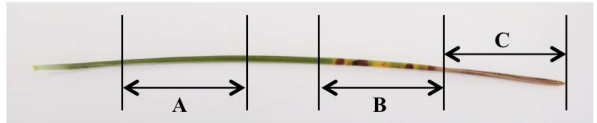

(a)

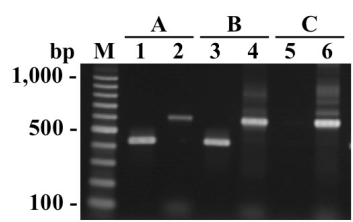

(b)
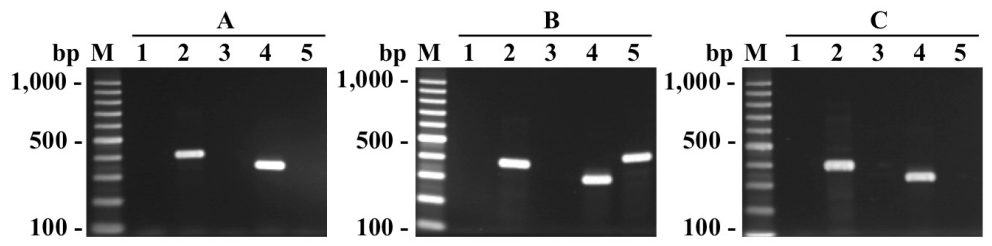

(c)

\begin{abstract}
Figure 3. Detection of phytopathogenic fungi from a needle (Sample 1). (a) Needle section (A to C) used for DNA extraction. (b) Electrophoretogram of the PCR products obtained in the first amplification of the DNA extracted from each needle section (A to C) in Figure 3(a). Lane 1, 3, and 5, rbcLF/rbcLR (RuBisCO large subunit gene of $P$. thunbergii); lane 2, 4, and 6, ITS1/ITS4 (fungal ITS-5.8S-ITS rDNA). (c) Electrophoretogram of the PCR products obtained by nested PCR amplification using the first amplification of the product and each specific primer. A, template from lane 2 of Figure 3(b); B, template from lane 4 of Figure 3(b); C, template from lane 6 of Figure 3(b). Lane 1, Rk1F/Rk1R (R. kalkhoffii); lane 2, Sp1F/Sp1R (S. pini-thunbergii); lane 3, Lo1F/Lo1R (L. conigenum); lane 4, Le1F/Le1R (L. acicola); lane 5, Pe1F/Pe1R (P. neglecta). M, 100 bp DNA ladder marker.
\end{abstract}

the withered region of a needle due to DNA degradation. In contrast, amplification products were obtained using ITS1/ITS4 to target the ITS-5.8S-ITS rDNA of fungi in samples A, B and C, indicating whether any fungi were present inside the needle. Furthermore, it was suggested that the fungal biomass of sample B and sample $\mathrm{C}$ would be greater than that of sample A because the amplification fragments of sample B and sample C were larger than that of sample A. Nested PCR revealed that S. pini-thunbergii and L. acicola were detected in all samples (A to C), whereas $P$. neglecta was only detected in sample B (Figure 3(c)). We further evaluated another needle (Figure 4). Amplification products were obtained using rbcLF/rbcLR to target the RuBisCO large subunit gene in sample D and sample E but not in sample F (Figure 4(b)). These results were almost the same except for sample $\mathrm{D}$, in which no amplification products were observed. It was noted that $S$. pini-thunbergii and $P$. neglecta were detected in sample D even with no amplification products being observed (Figure 4(c)). On the other hand, R. kalkhoffii, S. pini-thunbergii, L. acicola, and P. neglecta were detected in sample E and sample F (Figure 4(c)), indicating that these fungi can exist inside the withered region of a needle.

We tried to evaluate the possibility that these fungi could be detected from needles preserved in a freezer for one week (Figure 5). As a result, amplification products were obtained using the rbcLF/rbcLR primer pair to target the RuBisCO large subunit gene in sample $\mathrm{H}$ and sample I, while amplification products were obtained using the ITS1/ITS4 primer pair to target the ITS-5.8S-ITS rDNA of fungi in samples G, H and I. Nested PCR revealed that $R$. kalkhoffii, S. pini-thunbergii, $L$. conigenum, and $L$. acicola were detected in sample G, whereas $R$. kalkhoffii, S. pini-thunbergii, L. acicola and P. neglecta were detected in sample H and sample I (Figure 5(c)).

Finally, we tried to evaluate the detection of the endophytic and phytopathogenic fungi from healthy needles without any lesions. It was clearly demonstrated that amplification products were obtained using the rbcLF/rbcLR primer pair to target the RuBisCO large subunit gene, whereas no amplification products were visually observed using the ITS1/ITS4 primer pair to target the ITS-5.8S-ITS rDNA of fungi in all samples (Figure 6(b)). Nested PCR revealed that there was no amplification product using five specific primer pairs in sample $\mathrm{K}$ and sample $\mathrm{L}$, suggesting that $R$. kalkhoffii, S. pini-thunbergii, L. conigenum, L. acicola and P. neglecta were not present in the healthy needles in sample K and sample L (Figure 6(c)). Interestingly, nested PCR revealed that L. acicola and 


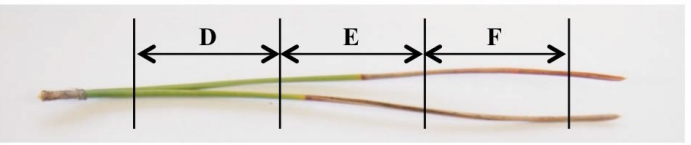

(a)

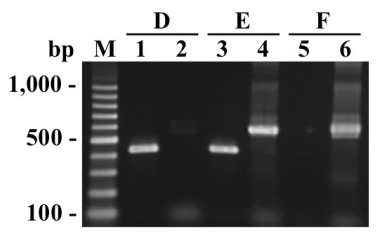

(b)
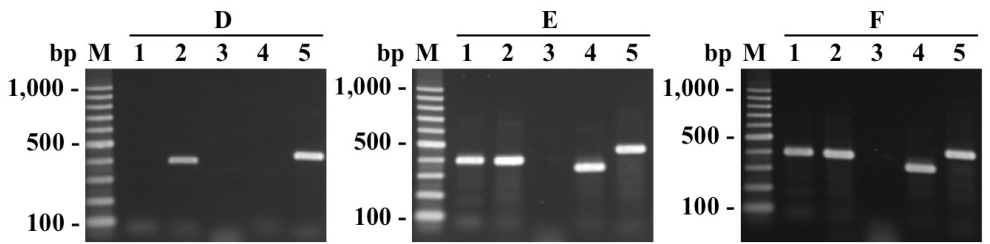

(c)

Figure 4. Detection of phytopathogenic fungi from a needle (Sample 2). (a) Needle section (D to F) used for DNA extraction; (b) Electrophoretogram of the PCR products obtained in the first amplification of the DNA extracted from each needle section (D to F) in Figure 4(a). Lane 1, 3, and 5, rbcLF/rbcLR (RuBisCO large subunit gene of $P$. thunbergii); lane 2, 4, and 6, ITS1/ITS4 (fungal ITS-5.8S-ITS rDNA); (c) Electrophoretogram of the PCR products obtained by nested PCR amplification using the first amplification of the product and each specific primer. D, template from lane 2 of Figure 4(b); E, template from lane 4of Figure 4(b); F, template from lane 6 of Figure 4(b). Lane 1, Rk1F/Rk1R (R. kalkhoffii); lane 2, Sp1F/Sp1R (S. pini-thunbergii); lane 3, Lo1F/Lo1R (L. conigenum); lane 4, Le1F/Le1R (L. acicola); lane 5, Pe1F/Pe1R (P. neglecta). M, 100 bp DNA ladder marker.

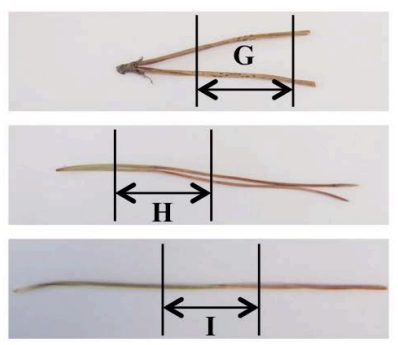

(a)

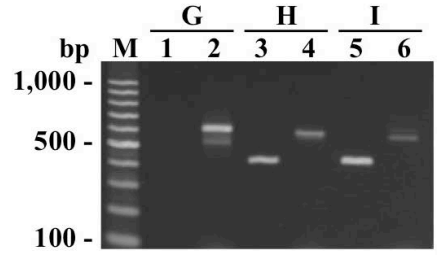

(b)
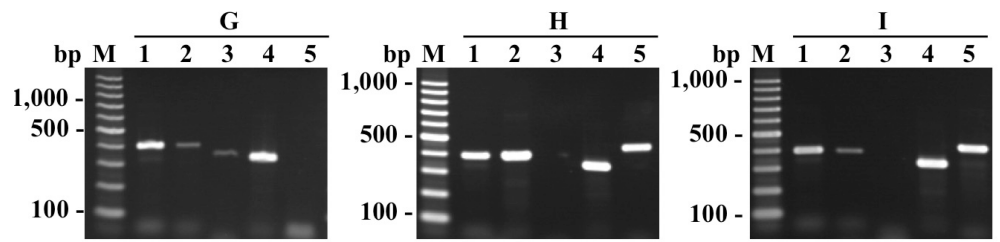

(c)

Figure 5. Detection of phytopathogenic fungi from a needle preserved in a freezer. (a) Needle section (G to I) used for DNA extraction; (b) Electrophoretogram of the PCR products obtained by the first amplification of the DNA extracted from each needle section (G to I) in Figure 5(a). Lane 1, 3, and 5, rbcLF/ rbcLR (RuBisCO large subunit gene of $P$. thunbergii); lane 2, 4, and 6, ITS1/ITS4 (fungal ITS-5.8S-ITS rDNA); (c) Electrophoretogram of the PCR products obtained by nested PCR amplification using the first amplification of the product and each specific primer. G, template from lane 2 of Figure 5(b); H, template from lane 4 of Figure 5(b) I, template from lane 6 of Figure 5(b). Lane 1, Rk1F/Rk1R (R. kalkhoffii); lane 2, Sp1F/Sp1R (S. pini-thunbergii); lane 3, Lo1F/Lo1R (L. conigenum); lane 4, Le1F/Le1R (L. acicola); lane 5, Pe1F/Pe1R (P. neglecta). M, 100 bp DNA ladder marker. 


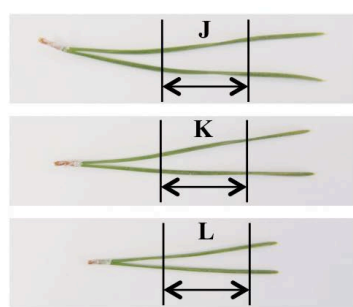

(a)

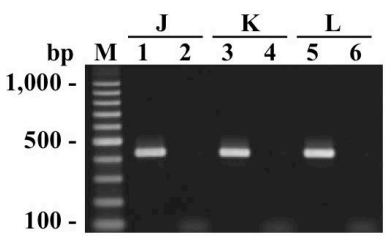

(b)

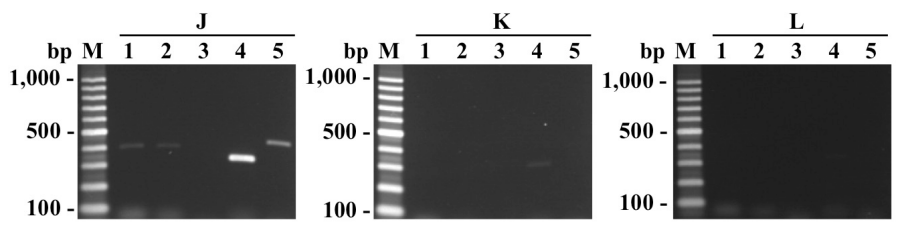

(c)

Figure 6. Detection of phytopathogenic fungi from a healthy needle. (a) Needle section (J to L) used for DNA extraction; (b) Electrophoretogram of the PCR products obtained in the first amplification of the DNA extracted from each needle section ( $\mathrm{J}$ to $\mathrm{L}$ ) in Figure 6(a). Lane 1, 3, and 5,rbcLF/rbcLR (RuBisCO large subunit gene of $P$. thunbergii); lane 2, 4, and 6, ITS1/ITS4 (fungal ITS-5.8S-ITS rDNA); (c) Electrophoretogram of the PCR products obtained by nested PCR amplification using the first amplification of the product and each specific primer. J, template from lane 2 of Figure 6(b); K, template from lane 4 of Figure 6(b); L, template from lane 6 of Figure 6(b). Lane 1, Rk1F/Rk1R (R. kalkhoffii); lane 2, Sp1F/Sp1R (S. pini-thunbergii); lane 3, Lo1F/Lo1R (L. conigenum); lane 4, Le1F/Le1R (L. acicola); lane 5, Pe1F/Pe1R (P. neglecta). M, 100 bp DNA ladder marker.

$P$. neglecta were only detected in sample J, suggesting that L. acicola and P. neglecta were present as endophytic fungi in the healthy needle in sample J (Figure 6(c)).

\section{Discussion}

The identification of endophytic and phytopathogenic fungi relied upon their culture-based morphological characteristics. The detection of these fungi by the traditional methods is time-consuming and requires extensive knowledge of fungal taxonomy. Recently, a variety of molecular tools have been used to differentiate fungal species from one another. Among these tools, species-specific polymerase chain reaction (PCR) has emerged as a powerful method of identifying and detecting fungi (Zhang et al., 2005; Broders \& Boland, 2010; Pravi et al., 2014; Popov et al., 2010). The development of PCR primers specific to the target organism is one of the most important steps in the PCR assay. The ITS regions of rDNA have been widely used to design specific primers for the identification of fungi of interest due to their high copy number and the fact that they contain both conserved and variable regions (Lovic et al., 1995; Ghignone et al., 2003; Stenström \& Ihrmark, 2005; Langrell, 2011; Lin et al., 2014). In addition, a large amount of rDNA sequence data from a variety of fungi is available in public databases. Analyses of these ITS sequences by means of multiple sequence alignment provide valuable information for the designation of species-specific PCR primer pairs. In this report, we demonstrated the detection of endophytic and phytopathogenic fungi from Japanese black pine needles with a PCR procedure using species-specific primers derived from the ITS region of the rDNA of these fungi. All of the primer pairs, Rk1F/ Rk1R, Sp1F/Sp1R, Lo1F/Lo1R, Le1F/Le1R, and Pe1F/Pe1R, were successful in specifically detecting $R$. kalkhoffii, S. pini-thunbergii, L. conigenum, L. acicola, and P. neglecta, respectively (Figure 2). Using these primer pairs, multiple fungi were detected not only in needles with lesions but also in healthy needles without symptoms (Figure 3, Figure 4, and Figure 6). Several kinds of spores with species-specific morphologies were sometimes observed on the same Japanese black pine needle after being incubated in a moist chamber (data not shown), indicating that several endophytic and phytopathogenic fungi can exist together on a single needle. Thus, the PCR-mediated detection developed in this study will be useful for evaluating the diversity of structure of the endophytic and phytopathogenic fungi on Japanese black pine needles without traditional culture methods.

Most of the phytopathogenic fungi on Japanese black pines seem to have a weak pathogenicity to the needles of these trees. Infection with Pestalotiopsis spp. was only induced on wounded needles, not on healthy needles 
(Takahashi \& Kobayashi, 1998), and the pathogenicities of $R$. kalkhoffiiand S. pini-thunbergii on the needles were rather weak under normal conditions (Tanaka \& Chiba, 1971; Kaneko et al., 1989), although L. acicola and Lophodermium spp. were observed to have pathogenicity on healthy Japanese black pine needles (Suto \& Ougi, 1998; Seo et al., 2012; Yamamoto et al., 1964). On the other hand, these fungi have been reported to be endophytic fungi of conifers, including the Japanese black pine (Yoo \& Eom, 2012; Qadri et al., 2014; Ganley et al., 2004; Ganley \& Newcombe, 2006). Thus, these fungi, which are considered to be phytopathogenic fungi, can be considered endophytic fungi of Japanese black pine needles in the initial stage after infection and cause disease with some typical symptoms and reproductive structures such as stromata in the latter stages.

Some endophytic fungi are latent pathogens that cause disease under certain conditions (Begoude et al., 2011; Sakalidis et al., 2011; Stanosz et al., 2001), while others can enhance host performance by conferring resistance to pathogens (Ganley et al., 2008; Romeralo et al., 2015). In addition, some endophytic fungi in the needles of conifers have been reported to be decomposers of needle litter (Müller et al., 2001; Korkama-Rajala et al., 2008; Osono \& Hirose, 2011; Yuan \& Chen, 2014). In this study, we isolated L. conigenum, L. acicola, P. neglecta, R. kalkhoffii, and S. pini-thunbergii from the Japanese black pine. In the future, these fungi should be characterized in terms of the parasitic or symbiotic relationships between them and their host plants, although the fungi would be considered latent pathogens of the Japanese black pine.

The detection of endophytic and phytopathogenic fungi using the PCR procedure with species-specific primers in this study could not reveal the amount of each endophytic and phytopathogenic fungus that was present. Quantitative real-time PCR assay is a powerful tool for the rapid, specific, and sensitive detection and quantification of fungi (Malvick \& Impullitti, 2007). Furthermore, other latent endophytic and phytopathogenic fungi are likely to exist in the needles of the Japanese black pine. Comprehensive analysis of amplified fragments of the ITS-5.8S-ITS region in Japanese black pine needles could reveal the diversity of endophytic and phytopathogenic fungi that are present. Finally, further methods of diagnosis such as loop-mediated isothermal amplification (LAMP) (Rigano et al., 2014) should be developed to establish a more rapid, more sensitive, and easier procedure for detecting and identifying the endophytic and phytopathogenic fungi in the needles.

\section{Acknowledgements}

This work was supported in part by a research grant from the dean of the Faculty of Life and Environmental Science, Shimane University.

\section{References}

Begoude, B. A. D., Slippers, B., Wingfield, M. J., \& Roux, J. (2011). The Pathogenic Potential of Endophytic Botryosphaeriaceous Fungi on Terminalia Species in Cameroon. Forest Pathology, 41, $281-292$. http://dx.doi.org/10.1111/j.1439-0329.2010.00671.x

Broders, K. D., \& Boland, G. J. (2010). Molecular Diagnostic Assay for Detection of the Butternut Canker Pathogen Sirococcus clavigignenti-juglandacearum. Plant Disease, 94, 952-958. http://dx.doi.org/10.1094/PDIS-94-8-0952

Chan, P. (2014). Japanese Black Pine, Kuro Matsu. In P. Chan (Eds.), The Bonsai Bible (pp. 88-89). London: Mitchell Beazley, a Division of Octopus Publishing Group.

Choi, Y. W., Hyde, K. D., \& Ho, W. H. (1999). Single Spore Isolation of Fungi. Fungal Diversity, 3, 29-38.

Ganley, R. J., Brunsfeld, S. J., \& Newcombe, G. (2004). A Community of Unknown, Endophytic Fungi in Western White Pine. Proceedings of the National Academy of Sciences of the United States of America, 101, 10107-10112. http://dx.doi.org/10.1073/pnas.0401513101

Ganley, R. J., \& Newcombe, G. (2006). Fungal Endophytes in Seeds and Needles of Pinus monticola. Mycological Research, 110, 318-327. http://dx.doi.org/10.1016/j.mycres.2005.10.005

Ganley, R. J., Sniezko, R. A., \& Newcombe, G. (2008). Endophyte-Mediated Resistance against White Pine Blister Rust in Pinus monticola. Forest Ecology and Management, 255, 2751-2760. http://dx.doi.org/10.1016/j.foreco.2008.01.052

Ghignone, S., Tamietti, G., \& Girlanda, M. (2003). Development of Specific PCR Primers for Identification and Detection of Rhizopycnis vagum. European Journal of Plant Pathology, 109, 861-870. http://dx.doi.org/10.1016/j.foreco.2008.01.052

Goh, T. K. (1999). Single-Spore Isolation Using a Hand-Made Glass Needle. Fungal Diversity, 2, 47-63.

Hata, K., \& Futai, K. (1995). Endophytic Fungi Associated with Healthy Pine Needles and Needles Infested by the Pine Needle Gall Midge, Thecodiplosis japonensis. Canadian Journal of Botany, 73, 384-390.

http://dx.doi.org/10.1139/b95-040 
Ito, K., \& Zinno, Y. (1972). Preliminary Information about Dothistroma Needle Blight of Pines in Japan. Forest Protection, 21, 86-89. (In Japanese)

Ito, K., Zinno, Y., \& Suto, Y. (1975). Dothistroma Needle Blight of Pines in Japan. Bulletin of the Government Forest Experiment Station, No. 272, 123-140.

Kaneko, S., Fujioka, H., \& Zinno, Y. (1989). A New Species of Septoria on Japanese Black Pine. Transactions of the Mycological Society of Japan, 30, 463-466.

Korkama-Rajala, T., Müeller, M. M., \& Pennanen, T. (2008). Decomposition and Fungi of Needle Litter from Slow- and Fast-Growing Norway Spruce (Picea abies) Clones. Microbial Ecology, 56, 76-89. http://dx.doi.org/10.1007/s00248-007-9326-y

Langrell, S. R. H. (2011). Nested Polymerase Chain Reaction-Based Detection of Dothistroma septosporum, Red Band Needle Blight of Pine, a Tool in Support of Phytosanitary Regimes. Molecular Ecology Resources, 11, 749-752. http://dx.doi.org/10.1111/j.1755-0998.2011.02996.X

Lin, Z., Xu, S., Que, Y., Wang, J., Comstock, J. C., Wei, J., McCord, P. H., Chen, B., Chen, R., \& Zhang, M. (2014). Species-Specific Detection and Identification of Fusarium Species Complex, the Causal Agent of Sugarcane Pokkah Boeng in China. PloS ONE, 9, e104195. http://dx.doi.org/10.1371/journal.pone.0104195

Lovic, B. R., Martyn, R. D., \& Miller, M. E. (1995). Sequence-Analysis of the Its Regions of rDNA in Monosporascus spp. to Evaluate Its Potential for PCR-Mediated Detection. Phytopathology, 85, 655-661. http://dx.doi.org/10.1094/Phyto-85-655

Malvick, D. K., \& Impullitti, A. E. (2007). Detection and Quantification of Phialophora gregata in Soybean and Soil Samples with a Quantitative, Real-Time PCR Assay. Plant Disease, 91, 736-742. http://dx.doi.org/10.1094/PDIS-91-6-0736

Min, Y. J., Park, M. S., Fong, J. J., Quan, Y., Jung, S., \& Lim, Y. W. (2014). Diversity and Saline Resistance of Endophytic Fungi Associated with Pinus thunbergii in Coastal Shelterbelts of Korea. Journal of Microbiology and Biotechnology, 24, 324-333. http://dx.doi.org/10.4014/jmb.1310.10041

Müller, M. M., Valjakka, R., Suokko, A., \& Hantula, J. (2001). Diversity of Endophytic Fungi of Single Norway Spruce Needles and Their Role as Pioneer Decomposers. Molecular Ecology, 10, 1801-1810. http://dx.doi.org/10.1046/j.1365-294X.2001.01304.x

Osono, T., \& Hirose, D. (2011). Colonization and Lignin Decomposition of Pine Needle Litter by Lophodermium pinastri. Forest Pathology, 41, 156-162. http://dx.doi.org/10.1111/j.1439-0329.2010.00648.x

Popov, A. P., Tsvetkov, I. L., Belov, A. A., Konichev, A. S., Ivanushkina, N. E., Kochkina, G. A., \& Ozerskaya, S. M. (2010). Molecular Genetic Identification of the Phytopathogenic Fungus Cryphonectria parasitica. Microbiology, 79, 223228. http://dx.doi.org/10.1134/S0026261710020141

Pravi, V., Jeeva, M. L., \& Archana, P. V. (2014). Rapid and Sensitive Detection of Sclerotium rolfsii Associated with Collar Rot Disease of Amorphophallus paeoniifolius by Species-Specific Polymerase Chain Reaction Assay. Molecular Biotechnology, 56, 787-794. http://dx.doi.org/10.1007/s12033-014-9757-x

Qadri, M., Rajput, R., Abdin, M. Z., Vishwakarma, R. A., \& Riyaz-Ul-Hassan, S. (2014). Diversity, Molecular Phylogeny, and Bioactive Potential of Fungal Endophytes Associated with the Himalayan Blue Pine (Pinus wallichiana). Microbial Ecology, 67, 877-887. http://dx.doi.org/10.1007/s00248-014-0379-4

Rigano, L. A., Malamud, F., Orce, I. G., Filippone, M. P., Marano, M. R., Morais do Amaral, A., Castagnaro, A. P., \& Vojnov, A. A. (2014). Rapid and Sensitive Detection of Candidatus Liberibacter Asiaticus by Loop Mediated Isothermal Amplification Combined with a Lateral Flow Dipstick. BMC Microbiology, 14, 86. http://dx.doi.org/10.1186/1471-2180-14-86

Romeralo, C., Santamaría, O., Pando, V., \& Diez, J. J. (2015). Fungal Endophytes Reduce Necrosis Length Produced by Gremmeniella abietina in Pinus halepensis Seedlings. Biological Control, 80, 30-39. http://dx.doi.org/10.1016/j.biocontrol.2014.09.010

Sakalidis, M. L., Hardy, G. E. S., \& Burgess, T. I. (2011). Endophytes as Potential Pathogens of the Baobab Species Adansonia gregorii: A Focus on the Botryosphaeriaceae. Fungal Ecology, 4, 1-14. http://dx.doi.org/10.1016/j.funeco.2010.06.001

Sakuyama, T. (1993). Physiological Characteristics of Two Pine Needle Cast Fungi, Lophodermium iwatense and Lophodermium pinastri. Journal of the Japanese Forest Society, 75, 273-277. (In Japanese with English Abstract)

Seo, S. T., Park, M. J., Park, J. H., \& Shin, H. D. (2012). First Report of Brown Spot Needle Blight on Pinus thunbergii Caused by Lecanosticta acicola in Korea. Plant Disease, 96, 914. http://dx.doi.org/10.1094/PDIS-12-11-1080-PDN

Stanosz, G. R., Blodgett, J. T., Smith, D. R., \& Kruger, E. L. (2001). Water Stress and Sphaeropsis sapinea as a Latent Pathogen of Red Pine Seedlings. New Phytologist, 149, 531-538. http://dx.doi.org/10.1046/j.1469-8137.2001.00052.x

Stenström, E., \& Ihrmark, K. (2005). Identification of Lophodermium seditiosum and L. pinastri in Swedish Forest Nurseries Using Species-Specific PCR Primers from the Ribosomal ITS Region. Forest Pathology, 35, 163-172.

http://dx.doi.org/10.1111/j.1439-0329.2005.00398.x 
Suto, Y. (2000). Septoria pini-thunbergii: A Fungus Produced on Dead Needles of Pinus thunbergii and P. ponderosa. Applied Forest Science, 9, 163-164. (In Japanese)

Suto, Y., \& Ougi, D. (1998). Lecanosticta acicola, Causal Fungus of Brown Spot Needle Blight in Pinus thunbergii, New to Japan. Mycoscience, 39, 319-325. http://dx.doi.org/10.1007/BF02464015

Takahashi, K., \& Kobayashi, T. (1998). Pestalotia Diseases of Pinus spp. and Picea glehni Caused by Pestalotiopsis spp. Journal of Tree Health, 2, 9-15. (In Japanese)

Takahashi, K., \& Kobayashi, T. (1999). Pestalotia Diseases of Pinus spp. Caused by Pestalotiopsis spp. Journal of Tree Health, 3, 21-30. (In Japanese with English Abstract)

Tanaka, K., \& Chiba, O. (1971). On a Needle Blight of Pine Caused by Rhizosphaera kalkhoffii Bubak: Life History, Physiological Characteristics and Pathogenicity of the Causal Fungus. Journal of the Japanese Forestry Society, 53, $279-286$. (In Japanese)

Townsend, A. M., \& Kwolek, W. F. (1987). Relative Susceptibility of Thirteen Pine Species to Sodium Chloride Spray. Journal of Arboriculture, 13, 225-228.

Tsukahara, H., Kozlowski, T. T., \& Shanklin, J. (1985). Tolerance of Pinus densiflora, Pinus thunbergii, and Larix leptolepis Seedlings to $\mathrm{SO}_{2}$. Plant and Soil, 88, 385-397. http://dx.doi.org/10.1007/BF02197495

White, T. J., Bruns, T., Lee, S., \& Taylor, J. W. (1990). Amplification and Direct Sequencing of Fungal Ribosomal Rna Genes for Phylogenetics. In M. A. Innis, D. H. Gelfand, J. Sninsky, \& T. J. White (Eds.), PCR Protocols: A Guide to Methods and Applications (pp. 315-322). San Diego, CA: Academic Press. http://dx.doi.org/10.1016/B978-0-12-372180-8.50042-1

Yamamoto, M., Yasumori, H., \& Suto, Y. (1964). Studies on the Pine Needle Cast (1) on the Pathogens of Pine Needle Cast. Journal of the Japanese Forest Society, 46, 347-354. (In Japanese with English Abstract)

Yoo, J. J., \& Eom, A. H. (2012). Molecular Identification of Endophytic Fungi Isolated from Needle Leaves of Conifers in Bohyeon Mountain, Korea. Mycobiology, 40, 231-235. http://dx.doi.org/10.5941/MYCO.2012.40.4.231

Yuan, Z., \& Chen, L. (2014). The Role of Endophytic Fungal Individuals and Communities in the Decomposition of Pinus massoniana Needle Litter. PLoS ONE, 9, e105911. http://dx.doi.org/10.1371/journal.pone.0105911

Zhang, J. X., Fernando, W. G. D., \& Remphrey, W. R. (2005). Molecular Detection of Apiosporina morbosa, Causal Agent of Black Knot in Prunus virginiana. Plant Disease, 89, 815-821. http://dx.doi.org/10.1094/PD-89-0815

Zhu, J., Gonda, Y., Yu, L., Li, F., Yan, Q., \& Sun, Y. (2012). Regeneration of a Coastal Pine (Pinus thunbergii Parl.) Forest 11 Years after Thinning, Niigata, Japan. PLoS ONE, 7, e47593. http://dx.doi.org/10.1371/journal.pone.0047593 